\title{
PSEUDO-MONOTONICITY AND DEGENERATED OR SINGULAR ELLIPTIC OPERATORS
}

\author{
P. Drábek, A. Kufner and V. Mustonen
}

Using the compactness of an imbedding for weighted Sobolev spaces (that is, a Hardy-type inequality), it is shown how the assumption of monotonicity can be weakened still guaranteeing the pseudo-monotonicity of certain nonlinear degenerated or singular elliptic differential operators.' The result extends analogous assertions for elliptic operators.

\section{INTRODUCTION}

Let us consider the second order differential operator

$$
u \mapsto-\sum_{i=1}^{N} \frac{\partial}{\partial x_{i}} a_{i}(x, u(x), \nabla u(x))+a_{0}(x, u(x), \nabla u(x))
$$

where the functions $a_{i}(x, \eta, \xi)$ and $a_{0}(x, \eta, \xi)$, defined on $\Omega \times \mathbb{R} \times \mathbb{R}^{N}$ with $\Omega$ an open subset of $\mathbb{R}^{N}$, satisfy suitable regularity and growth assumptions.

One of the conditions which allow us to apply the theory of monotone mappings is the so-called Leray-Lions condition:

$$
\sum_{i=1}^{N}\left(a_{i}(x, \eta, \xi)-a_{i}(x, \eta, \widehat{\xi})\right)\left(\xi_{i}-\widehat{\xi}_{i}\right)>0
$$

for almost all $x \in \Omega$, all $\eta \in \mathbb{R}$ and all $\xi, \widehat{\xi} \in \mathbb{R}^{N}$ with $\xi \neq \widehat{\xi}$.

As was shown recently (see $[2,3]$ ), if the mapping $T$ from $W_{0}^{1, p}(\Omega)$ to $W^{-1, p^{\prime}}(\Omega)$ generated by the operator (1.1) satisfies a slightly weaker condition than (1.2), namely

$$
\sum_{i=1}^{N}\left(a_{i}(x, \eta, \xi)-a_{i}(x, \eta, \hat{\xi})\right)\left(\xi_{i}-\widehat{\xi}_{i}\right) \geqslant 0
$$

Received 27th January, 1998

The work of the first and second authors was supported by grants of the Ministry of Education of Czech Republic \#VS 97156, of the Grant Agency of Czech Republic \#\# 201/97/0395, 201/97/0744 and of the Grant Agency of the Czech Academy of Sciences \#A1019506.

Copyright Clearance Centre, Inc. Serial-fee code: 0004-9729/98 \$A2.00+0.00. 
for almost all $x \in \Omega$, all $\eta \in \mathbb{R}$ and all $\xi, \widehat{\xi} \in \mathbb{R}^{N}$, then its pseudo-monotonicity for certain lower order parts $a_{0}$ can be ensured, and thus, existence results for boundary value problems can be appropriately extended.

These results are concerned with elliptic operators where the Sobolev spaces $W^{1, p}(\Omega)$ with $1<p<\infty$ play an essential role. Recently, the theory of monotone mappings was extended also to degenerate and/or singular elliptic operators where the weighted Sobolev space $W^{1, p}(\Omega ; w)$ plays the role of the classical Sobolev space $W^{1, p}(\Omega)$ (for details, see, for example, [1]). The aim of this note is to show that also in this case the weaker condition (1.3) can be applied.

For simplicity, we shall deal with the operator (1.1) with no lower order part, that is, $u \mapsto A u$, where

$$
A u=-\sum_{i=1}^{N} \frac{\partial}{\partial x_{i}} a_{i}(x, u, \nabla u)
$$

and we shall follow the ideas developed in [2]. Of course, the theory can be extended to the operator (1.1) and even to higher order operators (in divergence form). This will be done in a forthcoming paper where also the necessity of (1.3) and other monotonicity properties will be discussed.

\section{BASIC ASSUMPTIONS}

Our basic space will be the weighted Sobolev space

$$
W^{1, p}(\Omega ; w)
$$

with $\Omega$ an open set in $\mathbb{R}^{N}, 1<p<\infty$, and $w$ a collection of weight functions on $\Omega: w=\left\{w_{i}(x) ; i=0,1, \ldots, N\right\}, w_{i}$ measurable and positive almost everywhere in $\Omega$ and satisfying the conditions

$$
w_{i} \in L_{\mathrm{loc}}^{1}(\Omega), w_{i}^{1-p^{\prime}} \in L_{\mathrm{loc}}^{1}(\Omega), p^{\prime}=\frac{p}{p-1}
$$

The space $W^{1, p}(\Omega ; w)$ will be normed by

$$
\|u\|_{p, w}=\left(\int_{\Omega}|u(x)|^{p} w_{0}(x) d x+\sum_{i=1}^{N} \int_{\Omega}\left|\frac{\partial u}{\partial x_{i}}\right|^{p} w_{i}(x) d x\right)^{1 / p}
$$

Since we shall deal with the Dirichlet problem, we shall use the space

$$
X:=W_{0}^{1, p}(\Omega ; w)
$$


defined as the closure of the set $C_{0}^{\infty}(\Omega)$ with respect to the norm (2.2), and we shall suppose that the expression

$$
\|\| u \|_{X}=\left(\sum_{i=1}^{N} \int_{\Omega}\left|\frac{\partial u}{\partial x_{i}}\right|^{p} w_{i}(x) d x\right)^{1 / p}
$$

is a norm on $X$ equivalent to the norm (2.2). The reader can find conditions on the weight $w$ which guarantee this fact in [1]. Notice that $\left(X, \mid\|\cdot\| \|_{X}\right)$ is a uniformly convex (and thus reflexive) Banach space.

The following assumption will play an important role in our considerations:

$\left(A_{1}\right)$ There exist a weight function $\omega$ on $\Omega$ and a parameter $q, 1<q<\infty$, such that the (Hardy) inequality

$$
\left(\int_{\Omega}|u(x)|^{q} \omega(x) d x\right)^{1 / q} \leqslant C\left(\sum_{i=1}^{N} \int_{\Omega}\left|\frac{\partial u}{\partial x_{i}}\right|^{p} w_{i}(x) d x\right)^{1 / p}
$$

holds for every $u \in X$ with a constant $C>0$ independent of $u$ and, moreover, the imbedding

$$
X \hookrightarrow L^{q}(\Omega ; \omega)
$$

expressed by the inequality (2.5) is compact.

As usual, we introduce the semilinear Dirichlet form

$$
a(u, v)=\sum_{i=1}^{N} \int_{\Omega} a_{i}(x, u, \nabla u) \frac{\partial v}{\partial x_{i}} d x
$$

and suppose that the "coefficients" $a_{i}(x, \eta, \xi)$ satisfy the following (growth) conditions:

$\left(A_{2}\right)$ Each $a_{i}(x, \eta, \xi)$ is a Carathéodory function, that is, measurable in $x$ for any fixed $\zeta=(\eta, \xi) \in \mathbb{R}^{N+1}$ and continuous in $\zeta$ for almost all fixed $x \in \Omega$. There exist a constant $C_{1}>0$ and a function $g \in L^{p^{\prime}}(\Omega)$ such that

$$
\left|a_{i}(x, \eta, \xi)\right| \leqslant C_{1} w_{i}^{1 / p}(x)\left[g(x)+\omega^{1 / p^{\prime}}(x)|\eta|^{q / p^{\prime}}+\sum_{j=1}^{N} w_{j}^{1 / p^{\prime}}(x)\left|\xi_{j}\right|^{p-1}\right]
$$

for almost all $x \in \Omega$, all $\zeta=(\eta, \xi) \in \mathbb{R}^{N+1}$ and $i=1,2, \ldots, N$. (Here $\omega$ and $q$ are the weight function and the parameter from $(2.5)$, respectively.) 
Under these conditions, the Dirichlet form $a(u, v)$ is well-defined and bounded on $X$, which can be easily seen by Hölder's inequality.

Hence, the mapping $T$ from the space $X$ into its dual $X^{*}$, induced by the operator $A$ via formula

$$
\langle T u, v\rangle=a(u, v) \quad \text { for } \quad u, v \in X
$$

(where the brackets $\langle\cdot, \cdot\rangle$ denote the duality between $X^{*}$ and $X$ ) is a bounded mapping (that is $T$ maps bounded sets in $X$ onto bounded sets in $X^{*}$ ).

Let us recall the definition of pseudo-monotonicity:

$A$ mapping $T: X \rightarrow X^{*}$ is called pseudomonotone if for any sequence $\left\{u_{n}\right\}$ in $X$ with $u_{n} \rightarrow u$, that is weakly, and $\lim \sup \left\langle T u_{n}, u_{n}-u\right\rangle \leqslant 0$, it follows that

$$
T u_{n} \rightarrow T u \text { and }\left\langle T u_{n}, u_{n}\right\rangle \rightarrow\langle T u, u\rangle
$$

\section{The main Result}

The main result of this note which allows us to extend the existence results derived, for example, in [1], is given in the following assertion.

ProposItION 1. Let $\left(A_{1}\right)$ be satisfied. Let the functions $a_{i}: \Omega \times \mathbb{R} \times \mathbb{R}^{N} \rightarrow \mathbb{R}$ satisfy $\left(A_{2}\right)$ and (1.3). Then the mapping $T$ defined by (2.9) is pseudomonotone.

Proof: Let $u_{n} \rightarrow u$ in $X$ and

$$
\lim \sup \left\langle T u_{n}, u_{n}-u\right\rangle \leqslant 0 .
$$

Then $\frac{\partial u_{n}}{\partial x_{i}} \rightarrow \frac{\partial u}{\partial x_{i}}$ in $L^{p}\left(\Omega ; w_{i}\right)$ and $u_{n} \rightarrow u$ in $L^{p}\left(\Omega ; w_{0}\right)$, that is

$$
\frac{\partial u_{n}}{\partial x_{i}} w_{i}^{1 / p} \rightarrow \frac{\partial u}{\partial x_{i}} w_{i}^{1 / p} \quad \text { and } \quad u_{n} w_{0}^{1 / p} \rightarrow u w_{0}^{1 / p} \quad \text { in } \quad L^{p}(\Omega), i=1,2, \ldots, N
$$

Since $T$ is bcunded we have

$$
T u_{n} \rightarrow h \text { in } X^{*}
$$

and due to $(2.8)$ also

$$
a_{i}\left(\bullet, u_{n}, \nabla u_{n}\right) \rightarrow h_{i} \quad \text { in } \quad L^{p^{\prime}}\left(\Omega ; w_{i}^{1-p^{\prime}}\right)=\left(L^{p}\left(\Omega ; w_{i}\right)\right)^{*}
$$

$(i=1,2, \ldots, N)$ for some subsequence, where the action of $h$ is given by

$$
\langle h, v\rangle=\sum_{i=1}^{N} \int_{\Omega} h_{i} \frac{\partial v}{\partial x_{i}} d x \text { for all } v \in X .
$$


It follows from (2.10) and (2.11) that

$$
\lim \sup \left\langle T u_{n}, u_{n}\right\rangle \leqslant\langle h, u\rangle .
$$

On the other hand, by (1.3)

$$
\sum_{i=1}^{N} \int_{\Omega}\left[a_{i}\left(x, u_{n}, \bar{v}\right)-a_{i}\left(x, u_{n}, \nabla u_{n}\right)\right]\left(v_{i}-\frac{\partial u_{n}}{\partial x_{i}}\right) d x \geqslant 0
$$

for all $\bar{v}=\left(v_{i}\right) \in \prod_{i=1}^{N} L^{p}\left(\Omega ; w_{i}\right)$. Hence

$$
\begin{aligned}
& \sum_{i=1}^{N} \int_{\Omega} a_{i}\left(x, u_{n}, \nabla u_{n}\right) \frac{\partial u_{n}}{\partial x_{i}} d x \\
&\left.+\sum_{i=1}^{N} \int_{\Omega} a_{i}\left(x, u_{n}, \bar{v}\right) \frac{\partial u_{n}}{\partial x_{i}} d x-\sum_{i=1}^{N} \int_{\Omega} a_{i}\left(x, u_{n}, \nabla u_{n}\right) v_{i}\right) v_{i} d x
\end{aligned}
$$

It follows from (2.12) that

$$
\sum_{i=1}^{N} \int_{\Omega} a_{i}\left(x, u_{n}, \nabla u_{n}\right) v_{i} d x \rightarrow \sum_{i=1}^{N} \int_{\Omega} h_{i} v_{i} d x
$$

as $n \rightarrow \infty$. The compactness of the imbedding (2.6) implies

$$
u_{n} \rightarrow u \text { in } L^{q}(\Omega ; \omega) .
$$

Hence (2.16) together with (2.8) yield

$$
a_{i}\left(x, u_{n}, \bar{v}\right) \rightarrow a_{i}(x, u, \bar{v}) \quad \text { in } \quad L^{p^{\prime}}\left(\Omega ; w_{i}^{1-p^{\prime}}\right)
$$

and so

$$
\begin{gathered}
\sum_{i=1}^{N} \int_{\Omega} a_{i}\left(x, u_{n}, \bar{v}\right) \frac{\partial u_{n}}{\partial x_{i}} d x \rightarrow \sum_{i=1}^{N} \int_{\Omega} a_{i}(x, u, \bar{v}) \frac{\partial u}{\partial x_{i}} d x \\
\sum_{i=1}^{N} \int_{\Omega} a_{i}\left(x, u_{n}, \bar{v}\right) v_{i} d x \rightarrow \sum_{i=1}^{N} \int_{\Omega} a_{i}(x, u, \bar{v}) v_{i} d x
\end{gathered}
$$

as $n \rightarrow \infty$ for all $\bar{v}=\left(v_{i}\right) \in \prod_{i=1}^{N} L^{p}\left(\Omega ; w_{i}\right)$. It follows from (2.13), (2.14), (2.15), (2.17) and (2.18) that

$$
\begin{aligned}
\sum_{i=1}^{N} \int_{\Omega} h_{i} \frac{\partial u}{\partial x_{i}} d x & \geqslant \limsup \sum_{i=1}^{N} \int_{\Omega} a_{i}\left(x, u_{n}, \nabla u_{n}\right) \frac{\partial u_{n}}{\partial x_{i}} d x \\
& \geqslant \sum_{i=1}^{N} \int_{\Omega} h_{i} v_{i} d x+\sum_{i=1}^{N} \int_{\Omega} a_{i}(x, u, \bar{v}) \frac{\partial u}{\partial x_{i}} d x-\sum_{i=1}^{N} \int_{\Omega} a_{i}(x, u, \bar{v}) v_{i} d x
\end{aligned}
$$


Consequently,

$$
\sum_{i=1}^{N} \int_{\Omega}\left[a_{i}(x, u, \bar{v})-h_{i}\right]\left(v_{i}-\frac{\partial u}{\partial x_{i}}\right) d x \geqslant 0 \quad \text { for all } \quad \bar{v}=\left(v_{i}\right) \in \prod_{i=1}^{N} L^{p}\left(\Omega ; w_{i}\right) .
$$

Setting $\bar{v}=\nabla u+t \bar{z}$ with $t>0$ and $\bar{z}=\left(z_{i}\right) \in \prod_{i=1}^{N} L^{p}\left(\Omega ; w_{i}\right)$, we have

$$
\sum_{i=1}^{N} \int_{\Omega}\left[a_{i}(x, u, \nabla u+t \bar{z})-h_{i}\right] z_{i} d x \geqslant 0 \quad \text { for any } \bar{z}=\left(z_{i}\right) \in \prod_{i=1}^{N} L^{p}\left(\Omega ; w_{i}\right) .
$$

By letting $t \rightarrow 0+$, we conclude that

$$
a_{i}(x, u(x), \nabla u(x))=h_{i}(x) \quad \text { almost everywhere in } \Omega, i=1,2, \ldots, N \text {. }
$$

Hence $T u=h$ in $X^{*}$ and $T u_{n} \rightarrow T u$ has been proved.

It remains to show that

$$
\left\langle T u_{n}, u_{n}\right\rangle \rightarrow\langle T u, u\rangle
$$

Since we already have by (2.13),

$$
\lim \sup \left\langle T u_{n}, u_{n}\right\rangle \leqslant\langle h, u\rangle=\langle T u, u\rangle,
$$

it suffices to show that

$$
\liminf \left\langle T u_{n}, u_{n}\right\rangle \geqslant\langle T u, u\rangle
$$

Indeed, as in (2.14) with $\bar{v}=\nabla u$,

$$
\begin{aligned}
& \lim \inf \left\langle T u_{n}, u_{n}\right\rangle=\liminf \sum_{i=1}^{N} \int_{\Omega} a_{i}\left(x, u_{n}, \nabla u_{n}\right) \frac{\partial u_{n}}{\partial x_{i}} d x \\
& \geqslant \sum_{i=1}^{N} \int_{\Omega} h_{i} \frac{\partial u}{\partial x_{i}} d x+\sum_{i=1}^{N} \int_{\Omega} a_{i}(x, u, \nabla u) \frac{\partial u}{\partial x_{i}} d x-\sum_{i=1}^{N} \int_{\Omega} a_{i}(x, u, \nabla u) \frac{\partial u}{\partial x_{i}} d x \\
& =\langle T u, u\rangle+\langle T u, u\rangle-\langle T u, u\rangle=\langle T u, u\rangle,
\end{aligned}
$$

and the assertion follows.

Using the proposition just derived, we get the following general existence result which is a modification of the corresponding existence theorems in $[1$, Chapter 2]:

THEOREM 1. Suppose that the operator $A$ from (1.4) satisfies (1.3), (2.8) and the ellipticity condition

$$
\sum_{i=1}^{N} a_{i}(x, \eta, \xi) \xi_{i} \geqslant c_{0} \sum_{i=1}^{N} w_{i}(x)\left|\xi_{i}\right|^{p}
$$

for almost all $x \in \Omega$ and all $(\eta, \xi) \in \mathbb{R}^{N+1}$. Let $f \in X^{*}$. Then there exists at least one weak solution $u \in X$ of the (homogeneous) Dirichlet problem:

$$
a(u, v)=\langle f, v\rangle \text { for every. } v \in X .
$$




\section{EXAMPLES AND REMARKS}

EXAMPLE 1. Let us consider the following special case

$$
a_{i}(x, \eta, \xi)=w_{i}(x)\left|\xi_{i}\right|^{p-1} \operatorname{sgn} \xi_{i}+w_{0}(x) A_{0}(\eta)
$$

$i=1,2, \ldots, N$, with $w_{i}(x)$ given weight functions $(i=0,1, \ldots, N)$.

(i) For simplicity, we shall suppose that the weight functions $w_{1}(x), \ldots, w_{N}(x)$ coincide:

$$
w_{i}(x)=w(x), x \in \Omega, i=1,2, \ldots, N .
$$

Then we can consider the Hardy inequality (2.5) in the form

$$
\left(\int_{\Omega}|u(x)|^{q} \omega(x) d x\right)^{1 / q} \leqslant C\left(\int_{\Omega}|\nabla u(x)|^{p} w(x) d x\right)^{1 / p}
$$

and the growth conditions (2.8) will be satisfied if we suppose that

$$
\left|a_{i}(x, \eta, \xi)\right| \leqslant C_{1} w^{1 / p}(x)\left[\omega^{1 / p^{\prime}}(x)|\eta|^{q / p^{\prime}}+w^{1 / p^{\prime}}(x)\left|\xi_{i}\right|^{p-1}\right],
$$

$x \in \Omega, i=1, \ldots, N$. For $a_{i}$ from (4.1), it means that we suppose

$$
\left|w_{0}(x) A_{0}(\eta)\right| \leqslant C_{1} w^{1 / p}(x) \omega^{1 / p^{\prime}}(x)|\eta|^{q / p^{\prime}} .
$$

This condition together with the compactness of the imbedding expressed by (4.3) allows us to use the results mentioned in Section 3.

Let us point out that the condition (4.4) shows the mutual behaviour of the growth of the term $w_{0}(x) A_{0}(\eta)$ (expressed in terms of $|\eta|^{q / p^{\prime}}$ ) and the degeneration and/or singularity (expressed in terms of the weight functions $\left.w_{0}, w, \omega\right)$. Also, the role of the parameters $p, q$ and weights $w, \omega$ appearing in (4.3) becomes more transparent.

(ii) In particular, let us use the special weight functions $w_{0}, w, \omega$ expressed in terms of the distance to the boundary $\partial \Omega$ : denote $d(x)=\operatorname{dist}(x, \partial \Omega)$ and set

$$
w(x)=d^{\lambda}(x), w_{0}(x)=d^{\lambda_{0}}(x), \omega(x)=d^{\mu}(x) .
$$

Condition (4.4) then reads as

$$
\left|A_{0}(\eta)\right| \leqslant d^{\lambda / p+\mu / p^{\prime}-\lambda_{0}}(x)|\eta|^{q / p^{\prime}} .
$$

In this case, the Hardy inequality

$$
\left(\int_{\Omega}|u(x)|^{q} d^{\mu}(x) d x\right)^{1 / q} \leqslant C\left(\int_{\Omega}|\nabla u(x)|^{p} d^{\lambda}(x) d x\right)^{1 / p}
$$


holds and the corresponding imbedding is compact provided $\Omega$ is a bounded domain satisfying the cone condition and

(a) for $1 \leqslant p \leqslant q<\infty$,

$$
\lambda \neq p-1, \frac{N}{q}-\frac{N}{p}+1 \geqslant 0, \frac{\mu}{q}-\frac{\lambda}{p}+\frac{N}{q}-\frac{N}{p}+1>0,
$$

(b) for $1 \leqslant q<p<\infty$,

$$
\lambda \in \mathbb{R}, \frac{\mu}{q}-\frac{\lambda}{p}+\frac{1}{q}-\frac{1}{p}+1>0 .
$$

Moreover, the conditions (4.7) or (4.8) are necessary and sufficient for the compactness (see [4, Theorems 19.17 and 19.22]).

For example, a comparison of (4.8) and (4.5) shows that in the case (b) above, we can have

$$
\lambda_{0}<\mu(1+1 / q-1 / p) .
$$

EXAMPLE 2. We consider again the functions $a_{i}$ from (4.1), but now we choose

$$
w_{i}(x)=w(x) \quad \text { for } \quad i=1,2, \ldots, N-1, \quad w_{N}(x) \equiv 0 .
$$

To prove that $T$ is pseudomonotone, we can work with the same space as in Example 1 and consider the Hardy inequality (4.3). The difference between this case and the case considered in Example 1 is in the monotonicity condition (1.2) and/or (1.3). While in the case of Example 1 we have

$$
\begin{aligned}
& \sum_{i=1}^{N}\left(a_{i}(x, \eta, \xi)-a_{i}(x, \eta, \widehat{\xi})\right)\left(\xi_{i}-\widehat{\xi}_{i}\right) \\
& \quad=w(x) \sum_{i=1}^{N}\left(\left|\xi_{i}\right|^{p-1} \operatorname{sgn} \xi_{i}-\left|\widehat{\xi}_{i}\right|^{p-1} \operatorname{sgn} \widehat{\xi}_{i}\right)\left(\xi_{i}-\widehat{\xi}_{i}\right)>0
\end{aligned}
$$

for almost all $x \in \Omega$ (since the weight function $w$ is positive almost everywhere in $\Omega$ ) and for all $\xi, \widehat{\xi} \in \mathbb{R}^{N}$ with $\xi \neq \widehat{\xi}$, in the case (4.9) we have

$$
\begin{aligned}
& \sum_{i=1}^{N}\left(a_{i}(x, \eta, \xi)-a_{i}(x, \eta, \hat{\xi})\right)\left(\xi_{i}-\widehat{\xi}_{i}\right) \\
& \quad=w(x) \sum_{i=1}^{N-1}\left(\left|\xi_{i}\right|^{p-1} \operatorname{sgn} \xi_{i}-\left|\widehat{\xi}_{i}\right|^{p-1} \operatorname{sgn} \hat{\xi}_{i}\right)\left(\xi_{i}-\widehat{\xi}_{i}\right) \geqslant 0
\end{aligned}
$$

and the last inequality cannot be strict since for $\xi \neq \widehat{\xi}$ with $\xi_{N} \neq \widehat{\xi}_{N}$ but $\xi_{i}=\widehat{\xi}_{i}$, $i=1,2, \ldots, N-1$, the corresponding expression is zero. 


\section{REFERENCES}

[1] P. Drábek, A. Kufner and F. Nicolosi, Quasilinear elliptic equations with degenerations and singularities, De Gruyter Series in Nonlinear Analysis and Applications 5 (Walter de Gruyter, Berlin, Heidelberg, New York, 1997).

[2] J.P. Gossez and V. Mustonen, 'Pseudomonotonicity and the Leray-Lions condition', Differential Integral Equations 6 (1993), 37-45.

[3] V. Mustonen, 'Quasimonotonicity and the Leray-Lions condition', in Theory and applications of nonlinear operators of acretive and monotone type, (A.G. Kartsatos, Editor), Lecture notes in pure and applied mathematics 178 (Marcel Dekker, New York, 1996), pp. 215-222.

[4] B. Opic and A. Kufner, Hardy-type inequalities, Pitman Research Notes in Mathematics Series 219 (Longman Scientific and Technical, Harlow, 1990).

Department of Mathematics

University of West Bohemia

Univerzitni 8

30614 Plzeñ

Czech Republic

Department of Mathematical Sciences

University of Oulu

Linnanmaa

90570 Oulu

Finland
Mathematical Institute

Czech Academy of Sciences

Žitná 25

11567 Praha 1

Czech Republic 